\title{
Impact of COVID-19 pandemic on gynecological oncology care: Glimpse into Association of Gynecological Oncologists of India (AGOI) perspective
}

Geetu Bhandoria ( $\sim$ doctor_071277@yahoo.co.in )

Command Hospital, Pune

\section{Shylasree TS}

Tata Memorial Hospital, Mumbai

\section{Prashant Bhandarkar}

Bhabha Atomic Research Centre Hospital, Mumbai

\section{Vijay Ahuja}

Manipal Comprehensive Cancer Centre, Bangalore

\section{Amita Maheshwari}

Tata Memorial Hospital, Mumbai

\section{Rupinder Sekhon}

Rajiv Gandhi Cancer Institute \& Research Centre, New Delhi

\section{Somashekhar SP}

Manipal Comprehensive Cancer Centre, Bangalore

\section{Research Article}

Keywords: COVID-19, pandemic, gynecological oncology care, survey, AGOI

Posted Date: April 30th, 2020

DOI: https://doi.org/10.21203/rs.3.rs-24626/v1

License: (c) (1) This work is licensed under a Creative Commons Attribution 4.0 International License. Read Full License 


\section{Abstract}

Purpose: The notorious COVID 19 pandemic has caused rapid and drastic changes in cancer care all over the world in 2020. This online survey aims to assess the extent to which the pandemic has affected cancer care in gynecological oncology amongst members of Association of Gynecological Oncologists of India (AGOI), a registered professional society, founded in 1991.

Methods: We developed and administered a cross-sectional, flash survey to members of The Association of Gynecological Oncologists of India (AGOI) in the first week of April 2020. Data were analyzed using Microsoft Office Excel 2016. Results were expressed as percentages of total responses excluding blank or unattended response. Overall theme-specific responses were expressed as a spectrum of finding and related inferences were drawn.

Results: Among approached practitioners, 90 responded to the survey, more than $80 \%$ were practicing consultants and more than $50 \%$ from academic institutions. Results of the survey showed that the ongoing pandemic had severely affected gynecological oncology practice and care amongst all responders. There were modifications in diagnostic pathways, interventions and follow ups across all organ sites. There was near unanimous opinion on use of general safety measures to combat the virus and to use complete PPEs in high risk situation. There was mixed responses to alternative educational activities especially using electronic technology and distant learning methods. There was an optimism among responders with regards to the current situation normalizing in 3 to 6 months.

Conclusion: This study documents the pandemic affected scenario of gynecological cancer care and perceptions of Gynecological Oncologists in India. Significant effect on all aspects of cancer care were observed. Technological learning methods both for patient care and educational activities were being adopted by many responders.

Keywords: COVID-19, pandemic, gynecological oncology care, survey, AGOI

\section{Introduction}

On 31 December 2019, the WHO China Country Office was informed of cases of pneumonia of unknown etiology (unknown cause) detected in Wuhan City, Hubei Province of China. The causal agent was identified as a novel coronavirus (2019-nCOV) on $7^{\text {th }}$ January 2020. [1] 2019-nCOV was renamed as the SARS-Cov-2 virus and disease caused as COVID-19, most likely a zoonotic disease. [2,3] On 30 January 2020, following the recommendations of the Emergency Committee, the WHO Director-General declared that the outbreak constitutes a Public Health Emergency of International Concern (PHEIC) or pandemic. [4] There has been an unexpected and exponential rise in the number of cases all over the globe, with over 
1.8 million cases worldwide and more than 0.11 million fatalities. [5] The impact of COVID-19 is substantial and has already caused huge economic, social and health-care disruptions worldwide. [6-8]

\section{Methodology}

We conducted a cross-sectional self-administered online survey for this study purpose in first week of April 2020. The validated survey posed groups of questions on various challenges about management changes regarding gynecologic cancers, concerns about personal protection measures and social perspectives. The survey was piloted and refined based on feedback. Questions with ambiguous intent or options were refined. The interviews were carried out through online website www.surveymonkey.com. Whatsapp groups and personal mail addresses of members of AGOI were used. There were a set of closed and open-ended questions whose answers could be marked on a numerical scale. At the end of the survey period responses received were downloaded as a form of comma-separated values (CSV) file. Data were analyzed using Microsoft Office Excel 2016. Results were expressed as percentages of total responses excluding blank or unattended response. Overall theme-specific responses were expressed as a spectrum of finding and related inferences were drawn.

\section{Results}

We received 90 responses, among responders Gynecological oncologists were 61 (67.78\%); followed by Gynecologists 11 (12.22\%); then Surgical Oncologists 10 (11.11\%); and finally Medical Oncologists and Radiation Oncologists 4 responders each (4.44\%). Half of the responders $45(50 \%)$ were from the academic hospital; followed by the cancer centre 28 (31.11\%); then private hospitals with $14(15.56 \%)$ and $3(3.33 \%)$ from local or regional hospitals. $44(48.89 \%)$ of responders were consultants with experience of ten years or more; while 21 (23.33\%) were consultants with below ten years of experience; and 17 (18.89\%) were residents and $8(8.89 \%)$ were fellows.

The majority of responders $45(75 \%)$ told to have institutional or national guidelines, rest $15(25 \%)$ were told to follow international guidelines. $60 \%$ responder expressed about the lack of scientific evidence among guidelines. Responders were equally distributed when asked about the general management of gynecological cancer patients requiring surgery, as follows: Cancellation of most oncologic surgeries; Starting or prolonging the neo-adjuvant therapy; Surgical treatment in COVID-free centers and Surgical treatment delayed but already scheduled within next 3 months for all patients.

$58 \%$ of responders' institute has managed to install 'tele-consultation' services, while $16 \%$ told to have facility under process for the same. $44 \%$ of responders were told to have new cases based on institutional guidelines, $35 \%$ were seeing new cases but deferring assessment till lockdown is in effect and $21 \%$ were told to not have any new patients. $65 \%$ of responders were comfortable postponing definitive management of CIN-3 by 3 months, another $30 \%$ are still able to provide standard management. 
Early-stage cervical cancer: $34 \%$ of responders were able to continue with standard surgical management, $33 \%$ were offering chemo-radiation for all cases, rest were following less common options like neoadjuvant chemotherapy or radiotherapy. (Table 1)

Early-stage endometrial cancer: Almost half of the responders (48\%) were continuing standard surgery, rest were offering neoadjuvant hormonal therapy (37\% overall) or neoadjuvant chemotherapy followed by delayed surgery. (Table 1)

Early-stage vulvar cancer: $40 \%$ were observing these patients closely, $28 \%$ were continuing to offer standard surgery and rest (32\%) were offering neoadjuvant options with chemo or radiotherapy. (Table 1 )

The majority of responders (83-92\%) were continuing treating advanced stage gynecological cancers, though most of them have 'modified' standard management as per institutional protocols. (Figure 1)

$80 \%$ of responders felt strongly about 'cessation of elective benign surgeries' and $70 \%$ for 'cessation of elective cancer surgeries'. $68 \%$ of responders were not in favor of postponing delaying diagnostic services for 'new' patients. $82 \%$ strongly supported delaying or modifying standard 'follow-up protocols' of gynecological cancer patients.

$71 \%$ responders felt that elective benign surgeries should be postponed 'as long as necessary' to shift resources for COVID care. However, only $37 \%$ felt a delay of 2 weeks is acceptable for elective cancer surgeries and another $36 \%$ felt these surgeries could be delayed for 6 weeks. $52 \%$ felt the diagnosis of new patients could be delayed up to 2 weeks and $27 \%$ felt a delay of 6 weeks was acceptable.

Most responders have modified 'follow up' protocols, mostly with a 'less frequent' follow up approach. Patients with more than 5 years of disease-free survival were not being seen presently by $54 \%$ of responders. (Figure 2)

$75 \%$ of responders feel that full personal protection equipment (PPE) should be used only by those health care workers (HCW) who are managing suspected or confirmed COVID-19 patients. Another $22 \%$ feel that PPE should be used by all HCW in the current scenario. $92 \%$ responders believe that a combination of social distancing, face mask (any type) and hand hygiene are effective means to contain the pandemic.

$83 \%$ of responders felt that it will take 3-6 months for socio-cultural life to get back to normal. $37 \%$ felt about get back to standard cancer care in 6 weeks. Whereas $43 \%$ felt about required duration of 3 months to do so. $30 \%$ believed to resume surgical practice will start in 6 weeks, whereas $47 \%$ believed 3 months for the same. $68 \%$ believe that it will take 6 months or longer for surgical or scientific meetings to restart.

$95 \%$ responders felt strongly that 'cancellation of large scientific meets and sports events' are effective means to contain the viral spread. $95 \%$ felt the same for 'closing of borders and prohibition of travel'. $93 \%$ strongly support the 'lockdown of social and economic life'. Another $96 \%$ strongly support 'active contact tracing of possible infected persons' as a containment measure. $91 \%$ feel that there should be some penal action against those who dissent/desert above containment measures. $86 \%$ were comfortable with 
the cancellation of large public events 'as long as necessary'. 91\% were comfortable with the prohibition on traveling and closure of borders for 3 months to 'as long as necessary'. $88 \%$ of responders were comfortable with the closure of schools and universities for 3 months or longer. $52 \%$ feel that social and economic lockdown should continue as long as needed, another $24 \%$ were comfortable with the lockdown extending for 3 months. $84 \%$ felt that contact tracing of likely infected people should continue for 3 months or as long as needed.

Only $3 \%$ of responders told to have operated confirmed COVID case yet, while another $4 \%$ were awaiting results of the test by the time of survey. $22 \%$ of responders had taken Hydoxy-chloroquine (HCQ) prophylaxis at the time of answering the survey. $50 \%$ wish to take it but were waiting for stronger evidence to emerge for its efficacy, another $12 \%$ wish to take it but were concerned about cardiac toxicity and $16 \%$ don't want to take it at all.

Various alternative learning methods were suggested by responders as depicted in Figure 3. 54\% of responders felt that it would be the alternative learning methods were not good enough as there is 'less opportunity for professional interaction'. $42 \%$ were concerned about different geographic time zones during online events. $48 \%$ were concerned about acquiring new digital/social media skills to be part of elearning. And lastly $26 \%$ felt that there will less opportunity for networking in e-learning events

\section{Discussion}

AGOI largely consists of practicing Gyn-oncologists, gynecologists with special interest in Gyn- oncology or preventive oncology, surgical oncologists, medical and radiation oncologists. It was encouraging that two thirds of the responders (67\%) were practicing Gyn-oncologists. More than $80 \%$ of responders were from academic institutions or cancer centers.

One in two responders had an experience of more than 10 years which will give a realistic view of impact of the pandemic on clinical practice in gynecological oncology. At the time of survey, many centers and institutions in India were in the process of formulating their own guidelines/webinars or published guidelines which resembled the logistics of their working environment [9]. Hence two-third of responders were following some COVID cancer guidelines to manage gynecological cancers. [10-19] Bench to bedside research on COVID-19 was being generated fast track in the world making clinicians uncomfortable as they were not used to this speed. This might be the reasons that $60 \%$ responders expressed lack of scientific evidence among existing guidelines

In cervix, two thirds of the patients were treated with standard optimal therapy (surgery/chemo-radiation). $[20,21]$ One third were given neoadjuvant chemotherapy, to delay surgery, which is not the standard of care at present but is a valid alternative if resources are stretched [22]. With regards to early endometrial cancer, surgery is the main stay of treatment and preferably Minimally Invasive Surgery (MIS), [23] but only one in two received surgery. Some form of treatment was advocated (hormonal/NACT) so that surgery could be delayed. [24] There were issues with safety of Minimal access surgery during the initial part of pandemic with regards to smoke evacuation and intra-abdominal pressure which might have 
altered the clinician's decision to delay MIS. [25] Many early vulvar cancers were managed by observation and only a few received surgery or neoadjuvant therapy. Vulvar cancers constitute less than $10 \%$ of surgical workload, so interpretation is difficult as numbers treated during this time might be few. On speculation, elderly age group, delayed healing of the surgical wound and prolonged hospitalization might have shifted the balance towards observation with intent to delay surgery compared to cervix or endometrium. [26]

Amongst all intervention, in early stage gynecological cancers, surgery, when indicated appears to be the best possible solution where recovery from surgery is good and the probability of acquiring the virus is less in a screened patient. There is an increased risk of acquiring viral infection as Radiotherapy needs multiple visits to hospital and chemotherapy suppresses immunity and increases susceptibility to infection. $[13,14]$ There might have been obvious logistic reasons for following one treatment over the other, such as lack of availability of slots to treat, inability to travel to a center with RT or surgical facility or reduced number of medical/nursing staff working due to quarantine and lockdown issues. The overall oncological outcomes of early gynecological cancers is very good hence needs to be evaluated and compared to pre COVID literature as there are deviations in standard management during this pandemic all over the world. It remains to be seen how many were made susceptible to development of COVID-19 due to cancer related treatment and contributed to overall morbidity and mortality.

There is not much controversy in starting treatment for advanced gynecological cancers in the survey results. Most of these comprise of advanced ovarian cancers and neoadjuvant treatment is non inferior to extensive cytoreductive surgery. [27] There is a clause on performing extensive cytoreductive surgery (CRS) during the pandemic as intensive care beds need to be reserved preferably for COVID patients. Reduced blood donation camps leading to shortage of blood products also is a limiting factor for performing CRS with or without HIPEC. $[13,14]$. Increased susceptibility to the virus is a main concern in advanced ovarian cancer patients as they are generally over 60 years, frail, and immune suppressed during chemotherapy.[28-30]

More than $70 \%$ respondents felt that a delay in 2-6 weeks of active treatment of gynecological cancers is acceptable. This is in keeping with cancer guidance of referral to treatment of 30 day cutoff in most developed countries for starting active intervention from diagnosis and also the time frame for advocating adjuvant therapy. [14-16]

As expected, the response rate to either postponement or modifications in 'follow-up' of treated patients was high. Despite many years of discussion, there is always resistance to change traditional physical follow up methods all over the world. [31] Now, the pandemic has changed the way we work by incorporating telemedicine [32]. Whether this will permanently change the way we consult patients in future remains to be seen. [30] This appears to be a good learning period for tele-follow-ups for cancer survivors for many cancer centers in India. [9]

Most practitioners in the survey felt that general measures are effective in containing the pandemic in day to day practice and complete PPEs to be reserved to workers dealing with high risk patients. A 
common sense, rationed approach to the availability of resources like PPEs and the magnitude of this pandemic is being followed cautiously not only in India but all over the world [33,34]. There was mixed response to HCQ prophylaxis as the evidence and availability are an issue. [35,36]

Most responders were optimistic that both professional and personal life will be back to normal within 3 to 6 months. This attitude might be based on the time needed for containment of infection in Wuhan. [37] Understandably, nearly all the professionals in the survey felt that large scientific meetings/conferences and schools should not be held as it enforces the law of containing the pandemic and medical ethics of 'first do no harm'. As many are in the learning curve of technology during the pandemic and not sure of its effectiveness and overall impact on learning compared to traditional methods, the response was reasonable. [30]

As per GLOBOCAN estimates, India had 96,922 new cervical cancers, 36,170 new ovarian cancers and 13,328 new endometrial cancers in 2018. [38]Stage at presentation is the most important prognostic factor across all malignancies. The current pandemic is forcing a delay in cancer management due to several reasons cited above. This is likely to have adverse effect on overall cancer care. We may soon be facing a bi-modal peak of cancer deaths; the imminent spike of those with decimated immunity falling victim to Covid-19 and the latent toll on those whose treatments were de-intensified, delayed, or canceled altogether. To survive SARS-CoV-2 only to then succumb to an undertreated cancer would be a Pyrrhic victory. The acuteness of infection and the chronicity of malignancy are the Scylla and Charybdis between which oncologists and their patients must now chart a very cautious course indeed. [39]

\section{Conclusions}

These are unprecedented times for healthcare, even more so for the oncology community. We have to adapt to the requirements of patients, care givers and institutions, in consonance with the stage of ongoing pandemic. Multi-disciplinary tumor board discussions, keeping patients involved in decision making and adopting a safe-approach both for patients and health care workers, will be key to overcome the crisis. Based on the survey results and general guidance on good clinical practice and governance, we suggest following recommendations to improve outcomes in gynecological cancers during and after the pandemic.

1. Enable cancer centers/institutions to establish uniform COVID-cancer guidelines

2. Shared decision making involving patients, multidisciplinary teams and administrators.

3. National/regional policy measures and incentives to increase the capacity of resources across all cancer care specialties to take on the back log of cancer/precancer waiting lists.

4. Increasing surgical capacity to perform surgery on all early gynecological/precancers as the overall survival is excellent in this group. Indicated cases to be offered MIS, with safety protocols in place.

5. All professional bodies (surgery/radiation/medical oncology/palliative) to agree on uniform guidelines to treat advanced gynecological cancer and cancers with poor prognosis 
6. An added benefit of reducing the possibility of exposure to the virus by aggressively adopting ERAS protocols. [40]

7. Optimal diagnostic methods and Alternative follow-up protocols to avoid multiple visits to hospital to reduce exposure, travel and over burdening of hospital resources

8. Establish comprehensive 'pandemic response plan' emphasizing on staff training in 'donning and doffing of PPEs, emergency intubations, intensive care management training of COVID cases for all clinical specialties.

9. Alternative teaching/laboratory based programs for continued medical education and skills training for residents.

10. Psychological and other support systems for health professionals to function optimally.

\section{Declarations}

\section{Acknowledgement}

We would like to acknowledge Delia Cortes Guiral, Colo-rectal surgeon from Spain and her team, whose survey was adopted and expanded for this study.

Competing interests: The authors declare no competing interests.

Funding: The authors received no external funding for this research.

Ethics/Consent: There were no patients involved in this study and thus patient's consent is not applicable. It being a Survey based study, not Ethical clearance was obtained and needed.

\section{References}

1. World Health Organization. Corona virus disease 2019 (COVID-19): Situation report-1

2. Kristian G Andersen, Andrew Rambaut, W lan Lipkin, Edward C Holmes, and Robert F Garry. The proximal origin of sars-cov-2. Nature Medicine, pages 1-3, 2020.

3. European Centre for Disease Prevention and Control

4. World Health Organization. Corona virus disease 2019 (COVID-19): Situation report-11

5. World Health Organization. Corona virus disease 2019 (COVID-19): Situation report- 85

6. Verity, Robert, Lucy C Okell, Ilaria Dorigatti, Peter Winskill, Charles Whittaker, Natsuko Imai, Gina Cuomo-Dannenburg, et al. "Estimates of the Severity of Coronavirus Disease 2019: A Model-Based Analysis." The Lancet Infectious Diseases. Accessed April 3, 2020. https://doi.org/10.1016/S14733099(20)30243-7.

7. Martin Reeves, Philipp Carlsson-Szlezak, Kevin Whitaker, and Mark Abraham. 2020. Post-COVID Era. BCG Henderson Institute, viewed 05 Apr 2020, https://www.bcg.com/en-in/publications/2020/8ways-companies-can-shape-reality-post-covid-19.aspx 
8. Blair Nimmo. 2020. Business continuity in a COVID-19 world. KPMG, viewed 05 Apr 2020, https://home.kpmg/xx/en/home/insights/2020/03/business-continuity-in-a-covid-19-world.html

9. The Tata Memorial Centre COVID-19 Working Group. The COVID-19 pandemic and the Tata Memorial Centre response. Indian J Cancer 0000;00:00-00. doi 10.4103/ijc.IJC_250_20

10. Burki, Talha Khan. "Cancer Guidelines during the COVID-19 Pandemic." The Lancet Oncology. Accessed April 3, 2020. https://doi.org/10.1016/S1470-2045(20)30217-5

11. You, Benoit, Alain Ravaud, Anne Canivet, Gérard Ganem, Philippe Giraud, Rosine Guimbaud, Laure Kaluzinski, et al. "The Official French Guidelines to Protect Patients with Cancer against SARS-CoV-2 Infection." The Lancet Oncology. Accessed April 3, 2020. https://doi.org/10.1016/S14702045(20)30204-7

12. Burki, Talha Khan. "Cancer Care in the Time of COVID-19." The Lancet Oncology. Accessed April 3, 2020. https://doi.org/10.1016/S1470-2045(20)30201-1

13. BGCS framework for care of patients with gynaecological cancer during the COVID-19 Pandemic. British Gyn Cancer society. Final. 22 Mar 2020

14. Bhavana Pothuri, Angeles Alvarez Secord, Deborah Armstrong, John Chan, Warner Huh, Joshua Kesterson, Joyce Liu, Kathleen Moore, Amanda Nickles Fader, Shannon Westin, Wendel Naumann. Anti-cancer Therapy and Clinical Trial Considerations for Gynecologic Oncology Patients During the COVID-19 Pandemic Crisis: 03 Apr 2020. https://www.sgo.org/clinical-practice/covid-19communique/

15. Ramirez, Pedro T, Luis Chiva, Ane Gerda Z Eriksson, Michael Frumovitz, Anna Fagotti, Antonio Gonzalez Martin, Anuja Jhingran, and Rene Pareja. “COVID-19 Global Pandemic: Options for Management of Gynecologic Cancers." International Journal of Gynecologic Cancer, March 27, 2020, ijgc-2020-001419. https://doi.org/10.1136/ijgc-2020-001419.

16. Masumi Ueda, Renato Martins, Paul C. Hendrie, Terry McDonnell, Jennie R. Crews, Tracy L. Wong, Brittany McCreery, et al. "Managing Cancer Care During the COVID-19 Pandemic: Agility and Collaboration Toward a Common Goal.” Journal of the National Comprehensive Cancer Network J Natl Compr Canc Netw, 2020, 1-4.

17. Considerations on gynecologic cancer management during covid-19 pandemic. Brazilian society of surgical oncology statement. BSSO. 03 Apr 2020

18. COVID-19: Elective Case Triage Guidelines for Surgical Care. American College of Surgeons. 24 Mar 2020. https://www.facs.org/covid-19/clinical-guidance/elective-case

19. ANZGOG \& COVID-19: 31 March 2020 Statement. https://www.anzgog.org.au/anzgog-covid-19/

20. Shrivastava S, Mahantshetty U, Engineer R, et al. Cisplatin Chemoradiotherapy vs Radiotherapy in FIGO Stage IIIB Squamous Cell Carcinoma of the Uterine Cervix: A Randomized Clinical Trial. JAMA Oncol. 2018;4(4):506-513. doi:10.1001/jamaoncol.2017.5179

21. Datta NR, Stutz E, Liu M, et al. Concurrent chemoradiotherapy vs. radiotherapy alone in locally advanced cervix cancer: A systematic review and meta-analysis. Gynecol Oncol. 2017;145(2):374385. doi:10.1016/j.ygyno.2017.01.033 
22. Marchetti, C., Fagotti, A., Tombolini, V., Scambia, G., De Felice, F., Survival and toxicity in neoadjuvant chemotherapy plus surgery versus definitive chemoradiotherapy for cervical cancer: a systematic review and meta-analysis, Cancer Treatment Reviews Cancer Treatment Reviews (2019), doi: https://doi.org/10.1016/j.ctrv.2019.101945

23. NCCN Clinical Practice Guidelines in Oncology (NCCN Guidelines): Uterine neoplasms. Version 1.2020-06 Mar 2020. https://www.nccn.org/professionals/physician_gls/pdf/uterine.pdf

24. Dolmans, Marie-Madeleine, Matteo Lambertini, Kirsten Tryde Macklon, Teresa Almeida Santos, Ana Ruiz-Casado, Andrea Borini, Virginie Bordes, Lucy Frith, Ellen Van Moer, and Ariane Germeyer. "EUropean REcommendations for Female FERtility Preservation (EU-REFER): A Joint Collaboration between Oncologists and Fertility Specialists." Critical Reviews in Oncology/Hematology 138 (June 1, 2019): 233-40. https://doi.org/10.1016/j.critrevonc.2019.03.010.

25. AGES and EAES recommendations regarding surgical response to covid-19 crisis. Aurora Pryor.29 Mar 2020

26. Mert I, Cliby WA, Bews KA, Habermann EB, Dowdy SC. Evidence-based wound classification for vulvar surgery: Implications for risk adjustment. Gynecol Oncol. 2019;154(2):280-282. doi:10.1016/j.ygyno.2019.06.008

27. Coleridge SL, Bryant A, Lyons TJ, Goodall RJ, Kehoe S, Morrison J. Chemotherapy versus surgery for initial treatment in advanced ovarian epithelial cancer. Cochrane Database of Systematic Reviews 2019, Issue 10.Art. No.:CD005343. doi:10.1002/14651858.CD005343.pub4

28. Zhou, Fei, Ting Yu, Ronghui Du, Guohui Fan, Ying Liu, Zhibo Liu, Jie Xiang, et al. "Clinical Course and Risk Factors for Mortality of Adult Inpatients with COVID-19 in Wuhan, China: A Retrospective Cohort Study." The Lancet 395, no. 10229 (March 28, 2020): 1054-62. https://doi.org/10.1016/S01406736(20)30566-3.

29. Guan, Wei-jie, Zheng-yi Ni, Yu Hu, Wen-hua Liang, Chun-quan Ou, Jian-xing He, Lei Liu, et al. “Clinical Characteristics of Coronavirus Disease 2019 in China." New England Journal of Medicine, February 28, 2020. https://doi.org/10.1056/NEJMoa2002032

30. The Lancet Oncology. "COVID-19: Global Consequences for Oncology." The Lancet Oncology 21, no. 4 (April 1, 2020): 467. https://doi.org/10.1016/S1470-2045(20)30175-3.

31. Salani R, Khanna N, Frimer M, Bristow RE, Chen LM. An update on post-treatment surveillance and diagnosis of recurrence in women with gynecologic malignancies: Society of Gynecologic Oncology (SGO) recommendations. Gynecol Oncol. 2017;146(1):3-10. doi:10.1016/j.ygyno.2017.03.022

32. Hollander JE, Carr BG. Virtually Perfect? Telemedicine for Covid-19 [published online ahead of print, 2020 Mar 11]. N Engl J Med. 2020;10.1056/NEJMp2003539. doi:10.1056/NEJMp2003539

33. Fong MW, Gao H, Wong JY, et al. Nonpharmaceutical Measures for Pandemic Influenza in Nonhealthcare Settings-Social Distancing Measures [published online ahead of print, 2020 May 17]. Emerg Infect Dis. 2020;26(5):10.3201/eid2605.190995. doi:10.3201/eid2605.190995

34. Matrajt, Laura, and Tiffany Leung. "Evaluating the Effectiveness of Social Distancing Interventions against COVID-19." MedRxiv, January 1, 2020, 2020.03.27.20044891. 
https://doi.org/10.1101/2020.03.27.20044891

35. Information for Clinicians on Therapeutic Options for Patients with COVID-19. 11 Apr 2020. https://www.cdc.gov/coronavirus/2019-ncov/hcp/therapeutic-options.html

36. Liu, Jia, Ruiyuan Cao, Mingyue Xu, Xi Wang, Huanyu Zhang, Hengrui Hu, Yufeng Li, Zhihong Hu, Wu Zhong, and Manli Wang. "Hydroxychloroquine, a Less Toxic Derivative of Chloroquine, Is Effective in Inhibiting SARS-CoV-2 Infection in Vitro." Cell Discovery 6, no. 1 (March 18, 2020): 16. https://doi.org/10.1038/s41421-020-0156-0

37. Leung, Kathy, Joseph T Wu, Di Liu, and Gabriel M Leung. "First-Wave COVID-19 Transmissibility and Severity in China Outside Hubei after Control Measures, and Second-Wave Scenario Planning: A Modelling Impact Assessment." The Lancet. Accessed April 15, 2020.

https://doi.org/10.1016/S0140-6736(20)30746-7.

38. https://gco.iarc.fr/today/data/factsheets/populations/356-india-fact-sheets.pdf

39. Lewis, Mark A. "Between Scylla and Charybdis - Oncologic Decision Making in the Time of Covid19." New England Journal of Medicine, April 7, 2020. https://doi.org/10.1056/NEJMp2006588.

40. https://erassociety.org/eras-executive-committee-statement-regarding-surgery-and-anaesthesiaduring-the-covid-19-pandemic/

\section{Table 1}

Due to technical limitations Table 1 is available as a download in the Supplementary Files.

\section{Figures}

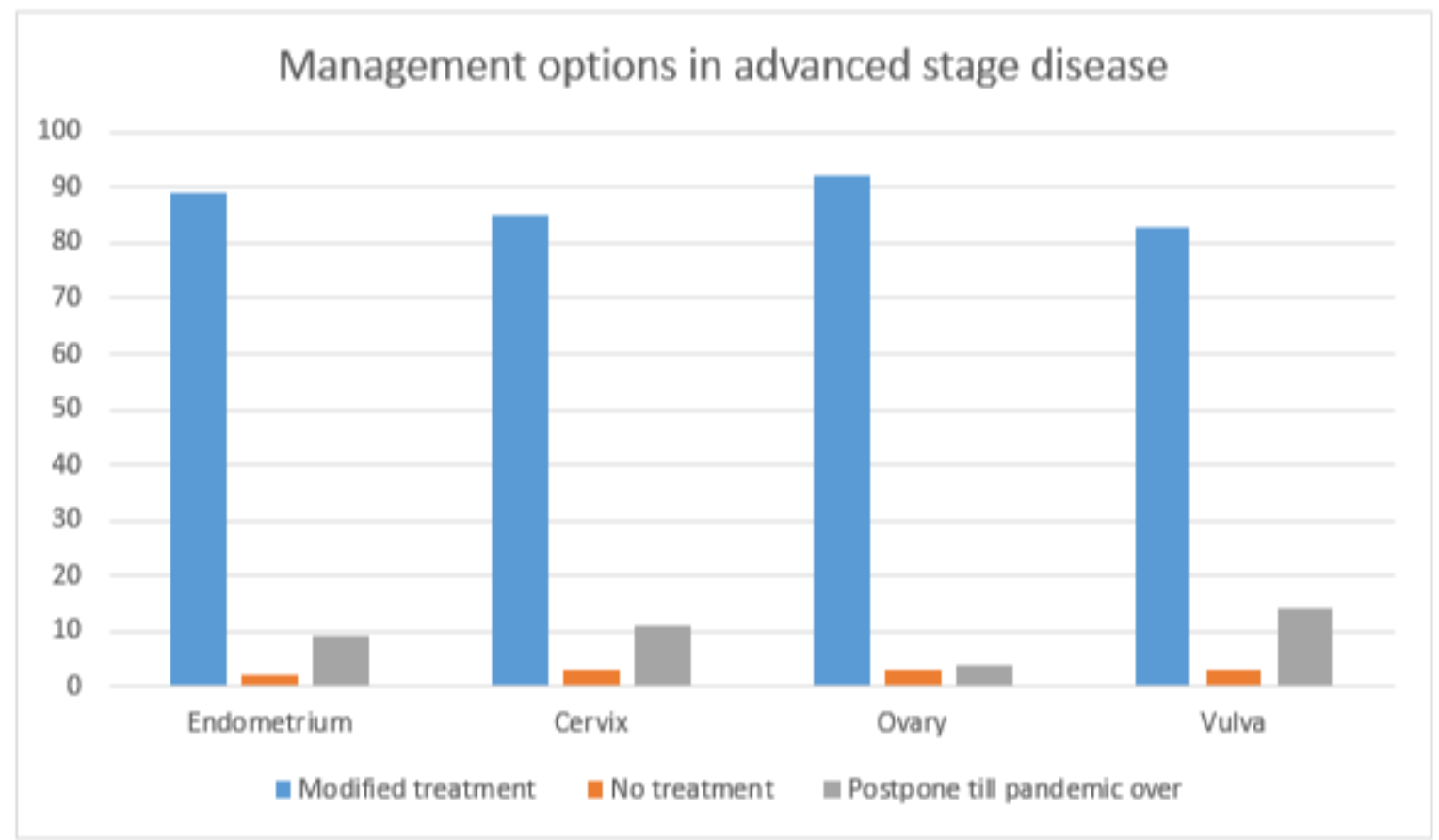


Management options for 'Advanced' stage disease

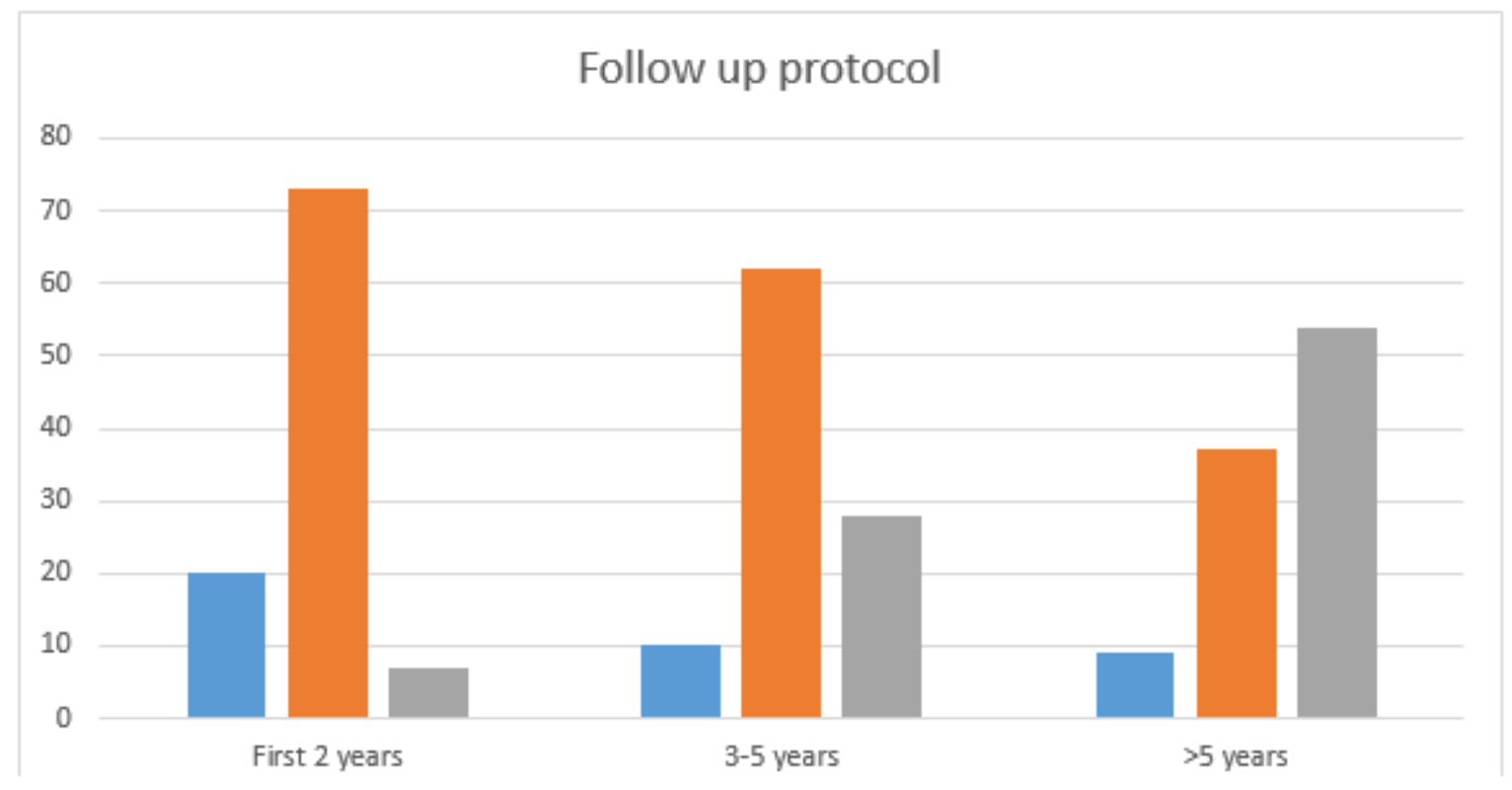

\section{Figure 2}

Suggested follow-up protocol, post treatment completion, weighing positive as well as negative consequences

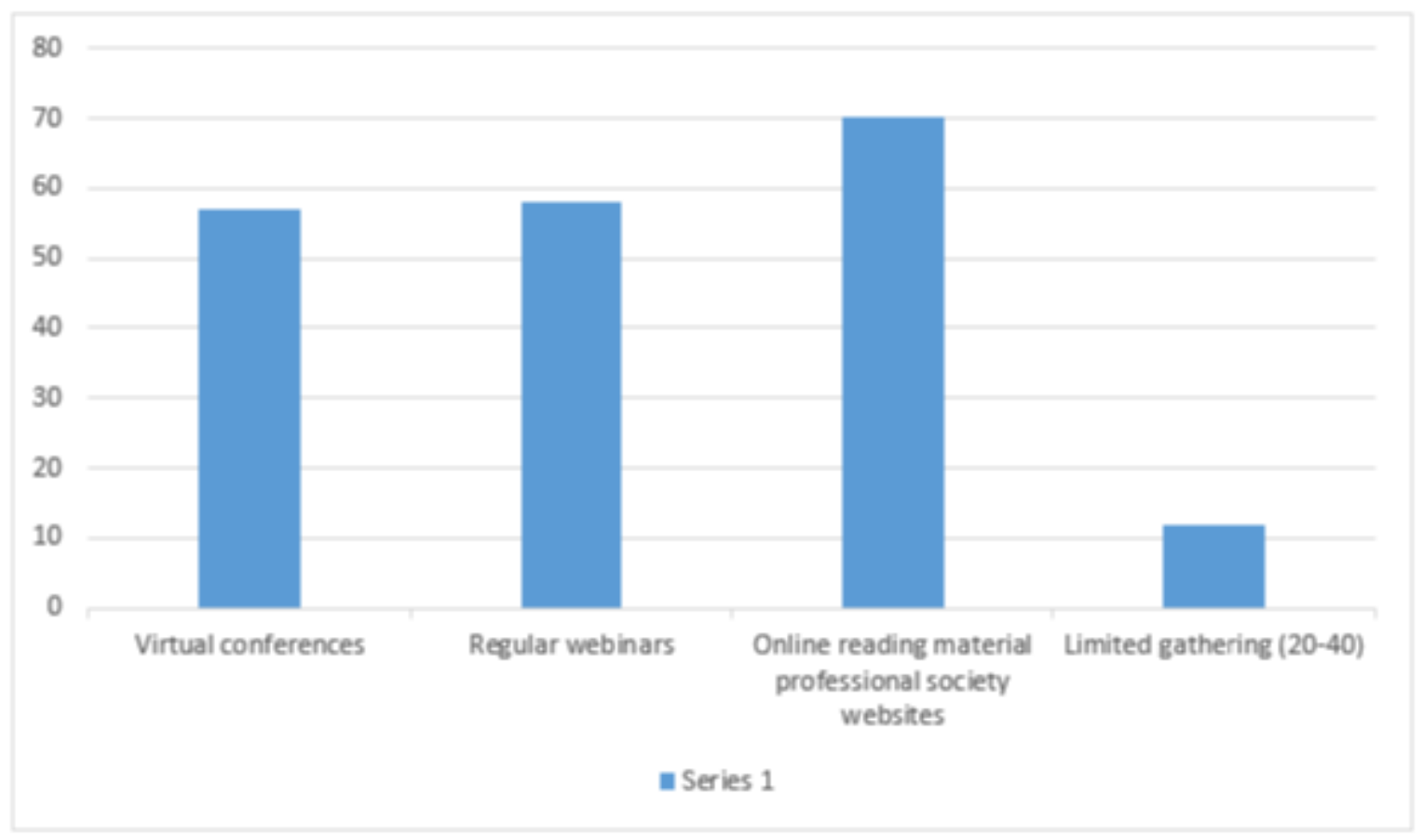

\section{Figure 3}

Alterative learning methods if lock-down/travel restrictions extends beyond 6 months

\section{Supplementary Files}


This is a list of supplementary files associated with this preprint. Click to download.

- COVIDsurvey.docx

- Table1.png 